\title{
Estimação do Volume de Eucaliptos por Krigagem e Cokrigagem no Semiárido Pernambucano
}

\author{
Wellington Jorge Cavalcanti Lundgren ${ }^{1}$, José Antônio Aleixo da Silva ${ }^{2}$, \\ Rinaldo Luiz Caraciolo Ferreira ${ }^{2}$ \\ ${ }^{1}$ Unidade Acadêmica de Serra Talhada - UAST, Universidade Federal Rural de Pernambuco -UFRPE, Serra Talhada/PE, Brasil \\ ${ }^{2}$ Universidade Federal Rural de Pernambuco - UFRPE, Recife/PE, Brasil
}

\begin{abstract}
RESUMO
Os volumes de 1875 árvores de um plantio de eucalipto foram rigorosamente cubadas pelo processo de Smalian, conhecendo-se assim o volume total do plantio que é de $166,14 \mathrm{~m}^{3}$. As árvores foram localizadas em um plano cartesiano $(\mathrm{X}, \mathrm{Y})$ e duas técnicas geoestatísticas foram aplicadas: a krigagem e cokrigagem. Para a cokrigagem foi utilizada como variável secundária o diâmetro a altura do peito (DAP), variável com alta correlação com o volume de madeira (Vol) do eucalipto. Três tamanhos de amostras foram retirados - 200, 100 e 50 -, e as estimativas dos volumes foram realizadas para cada uma das amostras usando as duas técnicas geoestatísticas. $\mathrm{O}$ tamanho da amostra não influiu nas estimativas do volume total do plantio. A cokrigagem forneceu melhores resultados do que a krigagem para as menores amostras. Os mapas da distribuição espacial do volume foram construídos.
\end{abstract}

Palavras-chave: manejo florestal, Caatinga, Polo Gesseiro, combustível e Araripe.

\section{Estimating of the Volume of Eucalyptus with Kriging and Cokriging in Semi-arid of Pernambuco}

\begin{abstract}
The volumes of 1875 eucalyptus plantation trees were strictly cubed by Smalian process, thus knowing the total volume of planting of $166.14 \mathrm{~m} 3$, the trees were located on a plane (X, Y) and two geostatistical techniques were applied, kriging and cokriging. For cokriging was used as the secondary variable $(\mathrm{DBH})$ diameter at breast height of the entire population, variable with high correlation with the volume of Eucalyptus wood. Three sizes of samples were taken - 200, 100 and 50 - and the estimates of the volumes were performed for each sample using both geostatistical techniques. The sample size did not affect the estimates of the total volume of planting. The cokriging provided better results than kriging for the smaller samples. The maps of spatial distribution volume were constructed.
\end{abstract}

Keywords: management of forest, Caatinga Conservation, Plaster Pole, fuel and Araripe. 


\section{INTRODUÇÃO}

Em todo o planeta, a madeira vem se tornando ano a ano um bem cada vez mais valioso, tanto pelas suas qualidades únicas como pelo aumento da dificuldade de ser conseguida na natureza. Biazus et al. (2010) fizeram um levantamento do preço médio de painéis de madeira no mercado mundial de 1997 até 2008 e mostram que entre 1997 e 2008 o valor aumentou de U\$ $400,00 / \mathrm{m}^{3}$ para U\$ $700,00 / \mathrm{m}^{3}$. Valverde et al. (2004) mostram que entre 2002 e 2004 o preço do metro estéril de madeira de eucalipto aumentou de $\mathrm{R} \$ 22,50$ para $\mathrm{R} \$ 45,00$. Miranda (2014) pesquisou sobre o custo para a produção de madeira em pé de eucalipto e o preço de mercado em algumas regiões do Brasil. De acordo com o autor, o preço máximo de venda alcançado em abril de 2014 foi em Palmas-TO, com U $\$ 27,68 / \mathrm{m}^{3}$.

A pressão da sociedade para que o desmatamento desenfreado seja extinto estimula o cultivo de árvores, o que torna a madeira mais cara do que a simplesmente coletada da mata nativa. Segundo a Advanced Financial Network (ADVFN, 2015), até o ano de 2014 o Brasil era o quarto maior produtor de celulose do mundo.

Em cultivos de florestas plantadas, o volume de madeira em pé é medida de difícil obtenção, e uma das técnicas para a obtenção desse atributo é a "cubagem rigorosa", que foi explicada e empregada por Paula et al. (2002). Quando o interesse é estimar o volume total ou individual de um plantio, é importante que o menor número possível de amostras seja de retiradas. Nogueira et al. (2000) afirmam que a geoestatística tem a vantagem de poder reduzir o número de amostras, se comparada a estatística clássica.

A geoestatística surgiu na década de sessenta do século XX, e Matheron (1963) explica com detalhes toda a teoria que envolve as técnicas krigagem e a cokrigagem. Essas técnicas se baseiam na existência da dependência espacial entre os atributos estudados. Na krigagem, o valor de um atributo (variável primária) é estimado através do valor de alguns desses atributos vizinhos ao que se deseja estimar. Na cokrigagem, o raciocínio é o mesmo, porém nessa técnica, além dos valores do atributo estudado próximos ao local onde o valor será estimado, é adicionado o valor de outro atributo correlacionado com o atributo desejado, chamado de variável secundária. Para que faça sentido o uso da técnica, é importante que a variável secundária seja de mais fácil medição que a variável primária e que possuam forte correlação entre si. A variável volume de madeira é de difícil obtenção e foi a variável primária. O DAP é uma medida de fácil obtenção e foi a variável secundária. O DAP é altamente correlacionado com o volume.

Poucas vezes a técnica geoestatística pôde ser testada com tanta segurança como nesse trabalho, pois toda a população de 1875 árvores foi derrubada e seus volumes rigorosamente medidos, de forma que é conhecido o volume total de madeira existente no plantio. As amostras foram retiradas depois da medição total, fornecendo a precisão das técnicas. A grande vantagem da geoestatística em ciência florestal é que com ela se pode estimar a variável no espaço, além da construção de mapas para o atributo, o que para manejo de florestas plantadas é uma informação de grande valor, pois saber onde exatamente se localizam as maiores ou as menores árvores de um plantio ajuda bastante em vários aspectos do manejo. Alguns autores já utilizaram a técnica para manejo de floresta, como Mello et al. (2009a), que utilizaram a geoestatística para estimar o volume de madeira e o número de fustes em um plantio de eucaliptos no munícipio de Avaré, no Estado de São Paulo.

Na técnica krigagem, apenas duas informações são necessárias para a estimação do volume de madeira: a localização da árvore no plantio, que será fornecida por um par ordenado $(\mathrm{X}, \mathrm{Y})$, e o valor do volume de madeira. A localização do atributo é fácil de ser obtida, pois o plantio tem espaçamento homogêneo. Para a cokrigagem seriam necessárias as mesmas informações, acrescidas da posição, também fácil de ser conseguidas da variável secundária e o próprio valor dessas variáveis conseguidas com a amostra. Neste trabalho será verificada a existência de dependência espacial para o volume de madeira e do DAP do eucalipto em uma floresta plantada, comparada com a estimação do volume de madeira pelas técnicas krigagem e cokrigagem e construídos os mapas através das duas técnicas.

O objetivo deste trabalho é estimar o volume total de madeira de eucalipto de uma floresta em pé com três quantidades de amostras diferentes - 200, 100 e 50 amostras - e comparar duas técnicas geoestatísticas - krigagem e cokrigagem - usadas para essa estimação, além 
de construir os mapas do volume pela krigagem e cokrigagem para os três diferentes tamanhos da amostra.

\section{MATERIAL E MÉTODOS}

A pesquisa foi realizada no ano de 2009 na Chapada do Araripe, que faz parte do Polo Gesseiro Pernambucano e possui coordenadas geográficas de $07^{\circ} 27^{\prime} 37^{\prime \prime}$ S e $40^{\circ} 24^{\prime} 36^{\prime \prime}$ W e altitude de 831 metros (Barros, 2009). O experimento denominado Módulo de Experimentação Florestal do Polo Gesseiro do Araripe está localizado na Estação Experimental do Instituto Agronômico de Pernambuco - IPA, situada no município de Araripina-PE e distante $23 \mathrm{~km}$ da cidade e foi implantado em março de 2002.

Um total de1875 árvores de eucaliptos com 7,5 anos de idade e distribuídos em 2,4 hectares com espaçamento de $3 \times 2 \mathrm{~m}$ foram derrubadas com motosserras e seus volumes medidos por cubagem rigorosa pelo método de Smalian, que é descrito por Paula et al. (2002). Todas as árvores foram localizadas em plano cartesiano por um par ordenado $(\mathrm{X}, \mathrm{Y})$, em que $\mathrm{X}$ e $\mathrm{Y}=$ distância em metros da árvore de origem $(0,0)$.

O plantio consistia de 15 diferentes clones de eucaliptos, e cada clone foi plantado em parcela de $7 \times 7$ árvores distribuídas em quatro blocos. Cada bloco continha duas repetições do mesma parcela, e na área existem espaços vazios que correspondem ao plantio já retirado de cinco espécies entre nativas e exóticas diferentes do eucalipto, com mesmo espaçamento e tamanho de parcela. Na Figura 1 é possível visualizar o esquema do experimento.

Três diferentes tamanhos de amostras do volume de madeira foram retiradas sistematicamente seguindo a orientação de $\mathrm{Y}$, em cada uma das amostras foram incluídas as árvores que formavam o limite do polígono do plantio. Os tamanhos das amostras foram 200, 100 e 50, todos os 1875 DAPs medidos foram utilizados como variável secundária para a cokrigagem. Na Figura 2, podemos visualizar a distribuição do local das árvores no plantio: em (a) a população de 1875 árvores, em (b) a amostra com 200 árvores, em (c) a amostra com 100 árvores e em (d) a amostra com 50 árvores. Os tamanhos das amostras foram escolhidos de forma que cada tamanho fosse exatamente a metade do anterior. O tamanho da amostra de 200 árvores foi escolhido arbitrariamente, pois foi considerado um bom tamanho (mais que $10 \%$ da população) para a variável de interesse, no caso o volume de madeira.

\subsection{Análise exploratória dos dados}

Mello (2004) afirma que com a normalidade dos dados são conseguidas características ótimas, porém distribuições não normais também podem fornecer bons resultados de estimação. Yamamoto \& Landim (2013) afirmam que transformações visando a normalidade só

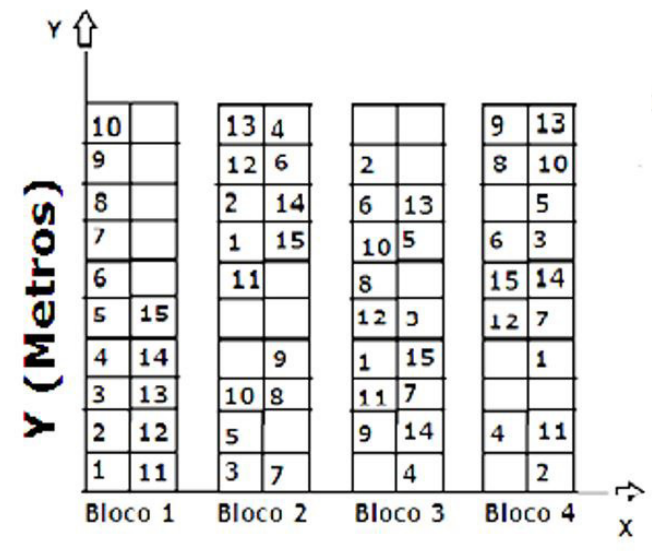

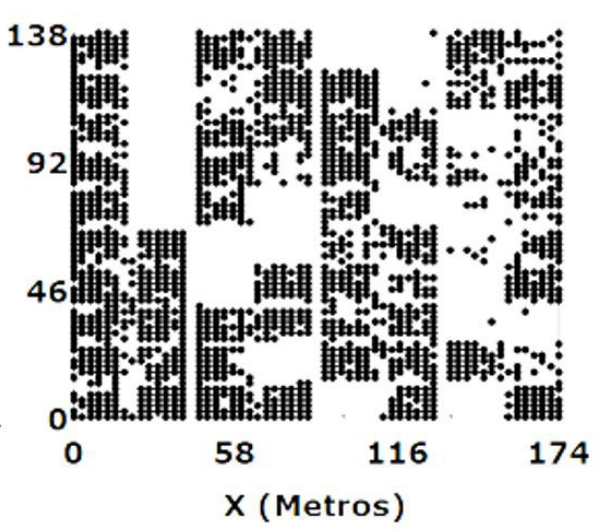

Figura 1. Esquema do experimento. Os números no esquema à esquerda representam os 15 diferentes tipos de clones. O esquema a direita representa a localização de cada árvore no plantio.

Figure 1. Experiment Scheme. The numbers in the left diagram representing 15 different clones. The scheme the right represents the location of each tree at planting. 

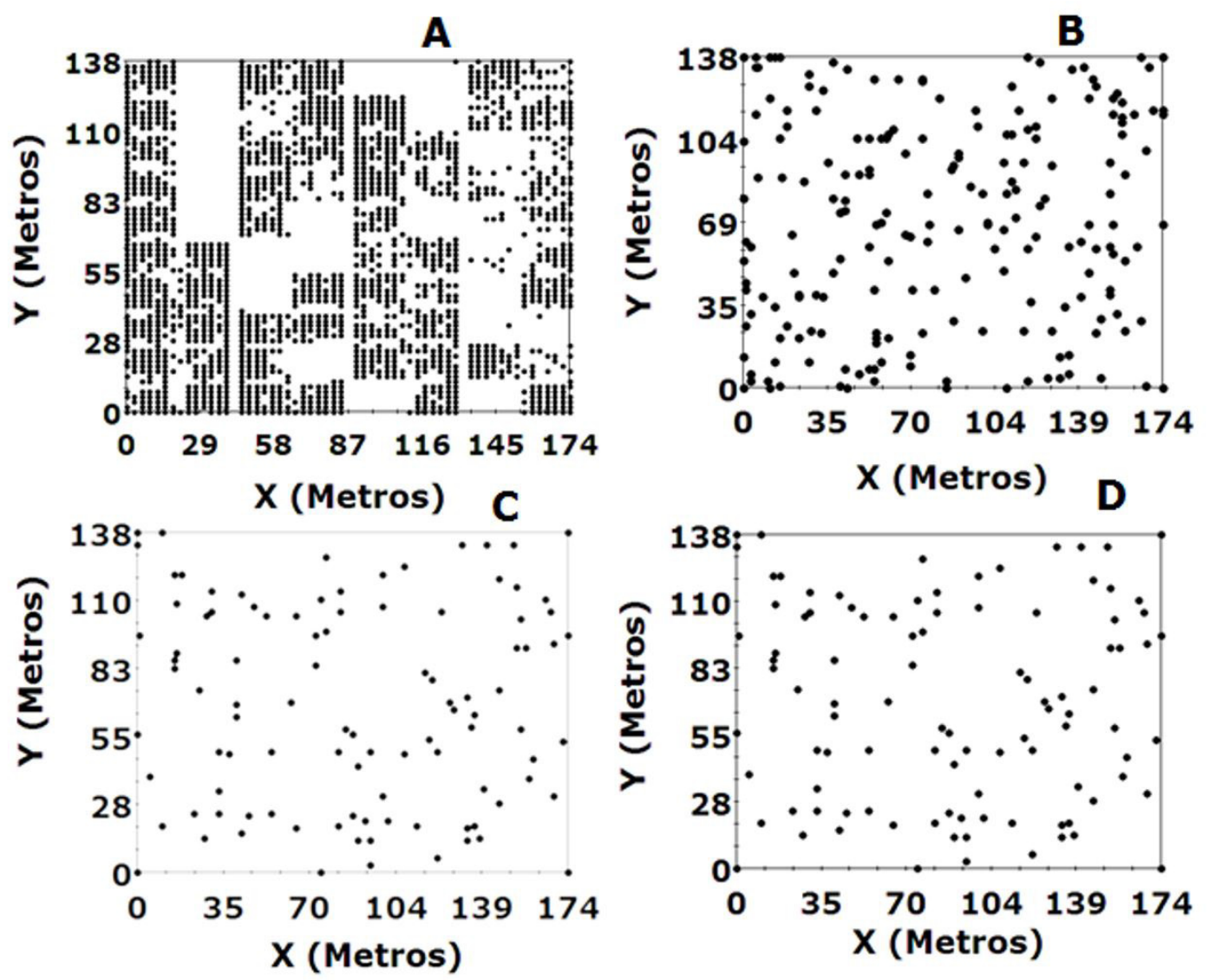

Figura 2. Distribuição populacional 1875 (A) e amostral de 200 (B), 100 (C) e 50 (D). Figure 2. Distribution population 1875 (A) and samples of 200 (B), 100 (C) and 50 (D).

devem ser tentadas se os dados apresentarem assimetria negativa. A normalidade dos dados foi testada pelo teste de Kolmogorov-Sminorf. No caso de não existir normalidade, foi utilizada a transformação logarítmica para tentar a normalização dos dados.

Foram construídos quatro semivariogramas amostrais nas direções $0^{\circ}, 45^{\circ}, 90^{\circ}$ e $135^{\circ}$ para cada uma das amostras, a fim de verificar a existência de anisotropia. Se os semivariogramas possuírem parâmetros próximos, é descartada a anisotropia.

Para a análise de tendência nos dados foram construídos dois gráficos, $\mathrm{X} \times$ Volume e $\mathrm{Y} \times$ Volume. Se os dados apresentarem estrutura organizada e o $\mathrm{R}^{2}$ for maior que 0,30 , estará confirmada a existência de tendência. Vieira (1995) afirma que esta deve ser retirada dos dados e o semivariograma deve ser ajustado para os resíduos. Para a cokrigagem, é necessário que as variáveis primária e secundária sejam fortemente correlacionadas, e é de conhecimento geral em ciências florestais o fato de que o DAP e o volume de madeira têm forte correlação, que foi calculada pela fórmula de Karl Pearson.

\subsection{Análise geoestatística dos dados}

Para cada uma das amostras do volume de madeira e para a população de DAPs, foi construído um semivariograma amostral simples usando a Equação 1.

$$
\Upsilon^{*}(h)=\frac{1}{2 \cdot N(h)} \sum_{i=1}^{N(h)}\left[Z\left(x_{i}\right)-Z\left(x_{i}+h\right)\right]^{2}
$$

em que: $\mathrm{Y}^{\star}(\mathrm{h})=$ semivariância estimada; $\mathrm{N}(\mathrm{h})$ = número de pontos separados pela distância $\mathrm{h} ; \mathrm{Z}\left(\mathrm{x}_{\mathrm{i}}\right)=$ valor do atributo no ponto $\mathrm{x}_{\mathrm{i}} ; \mathrm{Z}\left(\mathrm{x}_{\mathrm{i}}+\mathrm{h}\right)=$ valor do atributo no ponto distante $h$ do ponto $x_{\mathrm{i}}$. Os semivariogramas cruzados (Volume x DAP) foram construídos pela Equação 2.

$$
\Upsilon_{12}^{*}(h)=\frac{1}{2 N(h)} \sum_{i=1}^{N(h)}\left[Z_{1}\left(x_{i}\right)-Z_{1}\left(x_{i}+h\right)\right] \cdot\left[Z_{2}\left(x_{i}\right)-Z_{2}\left(x_{i}+h\right)\right]
$$


em que: $\mathrm{Y}^{*}(\mathrm{~h})=$ semivariância estimada; $\mathrm{N}(\mathrm{h})=$ número de pontos separados pela distância $h ; Z_{1}\left(X_{i}\right)=$ valor da variável primária no ponto $\mathrm{X}_{\mathrm{i}} ; \mathrm{Z}_{1}\left(\mathrm{X}_{\mathrm{i}}+\mathrm{h}\right)=$ valor da variável primária no ponto distante $\mathrm{h}$ do ponto $\mathrm{X}_{\mathrm{i}} ; \mathrm{Z}_{2}\left(\mathrm{X}_{\mathrm{i}}\right)=$ valor da variável primária no ponto $\mathrm{X}_{\mathrm{i}} ; \mathrm{Z}_{2}\left(\mathrm{X}_{\mathrm{i}}+\mathrm{h}\right)=$ valor da variável primária no ponto distante $\mathrm{h}$ do ponto $\mathrm{X}_{\mathrm{i}}$.

Para verificar e quantificar a existência de dependência espacial para os atributos dendrométricos adotados, foi utilizada a Equação 3:

$$
D E=\frac{\text { Patamar }- \text { Efeito Pepita }}{\text { Patamar }}
$$

Quanto maior o DE, maior será a dependência espacial do atributo.

Escolhidos os semivariogramas teóricos simples e cruzado, é possível, utilizando a krigagem e a cokrigagem, construir mapas do volume de madeira pelos dois processos. A Equação da krigagem 4 é apresentada a seguir.

$$
Z^{*}\left(x_{0}\right)=\sum_{i=1}^{N} \lambda_{i} Z\left(x_{i}\right)
$$

e a Equação da cokrigagem 5 é a que se segue.

$$
Z_{1}^{*}\left(x_{0}\right)=\sum_{j=1}^{N} \lambda_{1 j} Z_{1}\left(x_{1 j}\right)+\sum_{j=1}^{N} \lambda_{2 j} Z_{2}\left(x_{2 j}\right)
$$

em que: $Z^{\star}=$ valor estimado do atributo $Z ; x_{0}=$ local onde será estimado o valor da variável de interesse; $\mathrm{x}_{\mathrm{i}}=$ local do valor da variável de interesse; $\mathrm{x}_{1 \mathrm{j}}=$ local do valor da variável de primária; $\mathrm{x}_{2 \mathrm{j}}=$ local do valor da variável de secundária; $\lambda_{i}=$ pesos da interpolação; $\lambda_{1 \mathrm{i}}=$ pesos da interpolação para o atributo $Z_{1} ; \lambda_{2 \mathrm{i}}=$ pesos da interpolação para o atributo $Z_{2} ; Z_{1}^{*}=$ valor estimado do atributo $Z_{1}$ no ponto $x_{0} ; Z_{1}=$ valor medido do atributo $Z_{1}$ no ponto $x_{1 j} ; Z_{2}=$ valor medido do atributo $Z_{2}$ no ponto $x_{2 j}$.

Como o volume de madeira de cada eucalipto do plantio é conhecido, pois toda a população foi medida, o erro cometido em cada uma das estimativas será calculado pela Fórmula 6:

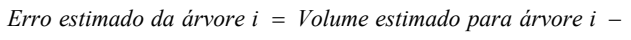

Serão construídos também os gráficos dos valores observados $\times$ valores estimados, e uma equação de regressão para cada gráfico será construída, os valores dos coeficientes angulares, do intercepto e do $\mathrm{R}^{2}$ serão comparados. O software GS+ foi utilizado para o cálculo das estatísticas clássicas, construção do mapa de localização das árvores no campo, para construção dos semivariogramas teóricos e amostrais e para a construção dos mapas pela krigagem e cokrigagem. A construção dos gráficos dos valores observados $\mathrm{x}$ valores estimados foi realizada pelo software Excel.

\section{RESULTADOS E DISCUSSÃO}

O volume total de madeira foi medido, fornecendo o valor de 166,14 metros cúbicos para uma área de 2,4 ha. O plantio forneceu uma média de $69,22 \mathrm{~m}^{3} / \mathrm{ha}$, valor bem abaixo do encontrado por Mello et al. (2006) em plantio comercial no município de Luiz Antônio-SP, onde sete talhões foram analisados e encontradas médias entre 205 e $273 \mathrm{~m}^{3} / \mathrm{h}$, uma diferença mínima de $135,78 \mathrm{~m}^{3} /$ ha em favor dos resultados de Mello. Vale ressaltar que no presente experimento 15 diferentes clones de eucaliptos foram testados para uma área dentro do semiárido pernambucano.

As estatísticas clássicas para o volume de madeira da população e de cada uma das amostras estão apresentadas na Tabela 1 . O coeficiente de correlação entre o DAP e o volume de madeira foi medido e forneceu o resultado de $\mathrm{r}=0,94$, valor que confirma o DAP como excelente variável secundária a ser utilizada na cokrigagem quando a variável primária for o volume de madeira. O coeficiente de variação foi considerado alto para as medidas de volume tanto na população como nas amostras. Bognola et al. (2008) encontraram CV $=24,8 \%$ para o IMA (Incremento Média Anual) em plantio de Pinus taeda L., valor que mostra que no atual trabalho a variabilidade é pelo menos duas vezes superior, e a alta variabilidade dificulta estimativas mais precisas.

\subsection{Krigagem}

Nem o volume nem o DAP apresentaram distribuição normal, então foi realizada uma transformação logarítmica, e para o volume a normalidade foi conseguida, porém para o DAP não. Mello et al. (2009b) encontraram normalidade para o volume de madeira por hectare e para o número de fuste por hectare no município de Avaré-SP. 
Tabela 1. Estatísticas clássicas populacional e amostral: volume de madeira $\left(\mathrm{cm}^{3}\right)$ e do DAP $(\mathrm{cm})$.

Table 1. Classical statistics for the population and samples of volume of wood $\left(\mathrm{cm}^{3}\right)$ DAP $\left(\mathrm{cm}^{3}\right)$.

\begin{tabular}{|c|c|c|c|c|c|}
\hline \multirow{3}{*}{ Estatísticas } & \multicolumn{5}{|c|}{ Tamanho da amostra } \\
\hline & 1875 & 200 & 100 & 50 & 1875 \\
\hline & \multicolumn{4}{|c|}{ Volumes } & DAP \\
\hline Transf & Não & $\log$ & $\log$ & $\log$ & $\log$ \\
\hline Normal & Não & Sim & Sim & Sim & Sim \\
\hline Média & 88.607 & 90.472 & 95.763 & 95.142 & 11,9 \\
\hline Mediana & 74.990 & 76.338 & 77.934 & 77.162 & 16,9 \\
\hline Desv Pad & 52.330 & 54.712 & 59.090 & 57.627 & 2,81 \\
\hline Variância & 38.433 .522 & 93.474 .997 & 91.720 .807 & 20.888 .704 & 7,87 \\
\hline Mínimo & 7.047 & 7.047 & 25.061 & 25.273 & 0,60 \\
\hline Máximo & 467.665 & 348.558 & 348.558 & 348.558 & 25,9 \\
\hline Assimet & 2,07 & 1,96 & 2,06 & 2,19 & 0,89 \\
\hline Curtose & 6,59 & 5,15 & 5,21 & 6,29 & 1,68 \\
\hline CV (\%) & 59,06 & 60,47 & 61,70 & 60,57 & 23,43 \\
\hline
\end{tabular}

$\mathrm{CV}$ = coeficiente de variação; $\mathrm{Vol}=$ volume de madeira; Desv Pad = Desvio-padrão; Transf = transformação; Assimet = Assimetria.

Tabela 2. Parâmetros dos semivariogramas Simples e Cruzados Anisotrópicos nas quatro direções para o Ln (Vol) e Ln (Vol) x Ln (DAP) para os três tamanhos de amostras.

Table 2. Parameters of semivariogram Simple and Anisotropic Crusaders in the four directions to Ln (Vol) and Ln (Vol) x Ln (DAP) for the three sample sizes.

\begin{tabular}{|c|c|c|c|c|c|c|c|c|c|c|c|c|}
\hline \multirow{3}{*}{ Amost } & \multicolumn{12}{|c|}{ Direções: $0^{\circ}, 45^{\circ}, 90^{\circ}, 135^{\circ}$} \\
\hline & \multicolumn{6}{|c|}{ Variogramas Simples } & \multicolumn{6}{|c|}{ Variogramas Cruzados } \\
\hline & EP & $\mathbf{P}$ & $>\mathbf{A}$ & $<\mathbf{A}$ & $\mathbf{R}^{2}$ & Mod & EP & $\mathbf{P}$ & $>\mathrm{A}$ & $<\mathbf{A}$ & $\mathbf{R}^{2}$ & Mod \\
\hline 200 & 0,25 & 0,76 & 647 & 647 & 0,27 & Lin & 1,23 & 3,78 & 644 & 594 & 0,27 & Lin \\
\hline 100 & 0,21 & 0,71 & 578 & 578 & 0,14 & Lin & 1,14 & 3,45 & 605 & 605 & 0,11 & Lin \\
\hline 50 & 0,14 & 0,73 & 386 & 386 & 0,15 & Lin & 0,73 & 3,76 & 345 & 345 & 0,13 & Exp \\
\hline
\end{tabular}

EP = Efeito Pepita; P = Patamar; $>$ A = Maior Alcance; $<\mathrm{A}=$ Menor Alcance; Mod = Modelo; Amost = Amostra; Lin = Linear; Exp $=$ Exponencial

Foram construídos os semivariogramas amostrais e teóricos nas quatro direções - $0^{\circ}, 45^{\circ}, 90^{\circ}$ e $135^{\circ}$ - para as amostras de 200, 100 e 50 árvores, e verificou-se a existência de dependência espacial e descartada a anisotropia para as três amostras. A anisotropia foi encontrada por Cunha et al. (2013), quando eles utilizaram a krigagem e a cokrigagem para construir mapa pluviométrico para o estado do Espírito Santo, em que a variável primária foi a precipitação e as variáveis secundárias foram duas: a altitude e a distância do mar. A variável primária apresentou anisotropia quando os semivariogramas foram construídos para os períodos secos e úmidos do ano.

Em pesquisa realizada com eucaliptos, Mello et al. (2005) encontraram isotropia para quatro atributos dendrométricos analisados por eles, a saber: volume médio por hectare, altura média dominante, diâmetro médio quadrático e área basal média por hectare. Nos Semivariogramas Simples Anisotrópicos para as quatro direções adotadas, os pontos apresentaram comportamentos semelhantes, fato que atesta a existência de isotropia. A dependência espacial existe, pois à medida que as distâncias aumentam, a semivariância também aumenta. A Tabela 2 apresenta os valores dos parâmetros do semivariogramas, e os valores foram exatamente iguais para cada uma das direções adotadas.

As análises de tendências dos dados foram realizadas tanto para o volume como para o DAP, e existência de tendência foi descartada.

Os parâmetros dos semivariogramas simples teóricos utilizados para a krigagem, para os três tamanhos de amostras, estão apresentados na Tabela 3. A dependência espacial foi captada para os três tamanhos de amostras.

Nota-se que com a diminuição do número de amostras de 200 para $100, \mathrm{R}^{2}$ para os semivariogramas diminuiu, porém com a diminuição de 100 para 50 , houve um pequeno aumento no $\mathrm{R}^{2}$. Os resultados sobre o $\mathrm{R}^{2}$ coincidem com os obtidos por Souza \& Souza 
Tabela 3. Parâmetros dos semivariogramas simples isotrópicos das amostras.

Table 3. Parameters of simple isotropic sample semivariogram.

\begin{tabular}{cccccccc}
\multirow{2}{*}{ Amostra } & \multicolumn{9}{c}{ Parâmetros } \\
\cline { 2 - 8 } & Modelo & Ef pep & Patamar & Alc & R $^{2}$ & DE \\
200 & Esférico & 0,127 & 0,311 & 35,5 & 0,90 & 0,59 \\
100 & Esférico & 0,044 & 0,268 & 23,7 & 0,59 & 0,83 \\
50 & Esférico & 0,039 & 0,248 & 44,9 & 0,63 & 0,84 \\
\hline
\end{tabular}

Ef pep = Efeito Pepita; Alc = Alcance; DE = Dependência Espacial.

(2011), em que os valores do $\mathrm{R}^{2}$ oscilaram para quatro tamanhos de amostras $(208,105,58$ e 24$)$ de teor de argila e de areia, mostrando não haver correlação entre o tamanho da amostra e o valor do $\mathrm{R}^{2}$ para a construção do semivariograma amostral.

O efeito pepita teve diminuição diretamente proporcional ao número de amostras, indicando que quanto mais distantes umas das outras as amostras, mais deficiente ficou a detecção dos microerros no cálculo da semivariância amostral. Esse resultado difere completamente dos resultados conseguidos por Souza \& Souza (2011), em que o valor do efeito pepita foi inversamente proporcional ao tamanho da amostra.

O patamar teve diminuição diretamente proporcional ao número de amostras, indicando que quanto menor a amostra, mais deficiente ficou a determinação da semivariância. Novamente os resultados do atual trabalho diferem completamente do trabalho de Souza \& Souza (2011), em que o patamar foi inversamente proporcional ao número de amostras.

O DE teve aumento inversamente proporcional ao número de amostras, resultado que não corresponde aos encontrados por Souza \& Souza (2011), em que os DEs foram diretamente proporcionais ao número de amostras.

\subsection{Cokrigagem}

Para a cokrigagem com os três diferentes tamanhos de amostras foram utilizados os modelos dos semivariogramas simples isotrópicos já encontrados anteriormente para uso na krigagem.

Para o atributo DAP foram utilizados todos os dados da população, exatamente 1875 árvores. Os semivariogramas do DAP com as 1875 árvores foram construídos nas quatro direções e foi verificada a existência de isotropia. Os semivariogramas teóricos anisotrópicos do DAP nas quatro direções são idênticos.
Os parâmetros dos modelos teóricos foram todos iguais: o modelo adotado foi o Linear, Efeito Pepita = 0,0434, Patamar $=0,1341$, Maior Alcance $=662,30$ e Menor Alcance $=548,40$ metros e o $\mathrm{R}^{2}=0,481$, a variável secundária DAP não apresentou anisotropia. Cunha et al. (2013) utilizaram a cokrigagem para estimação de precipitação tendo como variável secundária a altitude. Apesar de eles afirmarem que provavelmente os dados de altitude apresentariam tendência, eles não realizaram as análises e adotaram a isotropia com a alegação de que assim fariam para facilitar os cálculos.

Foram construídos também os semivariogramas cruzados isotrópicos do $[\mathrm{Ln}(\mathrm{Vol})] \times[\operatorname{Ln}(\mathrm{DAP})]$ para cada uma das amostras, e os gráficos estão apresentados na Figura 3. Os gráficos das análises de anisotropia para os semivariogramas cruzados para cada uma das amostras foram construídos, porém, não foram inclusos no atual trabalho. Eles não apresentaram anisotropia, e os valores dos parâmetros para as quatro direções podem ser vistos na Tabela 2 .

O semivariograma isotrópico foi construído e está apresentado na Figura 3D. Os parâmetros do semivariograma simples isotrópico teórico para o DAP foram: Efeito Pepita $=0,0273$, Patamar $=0,0547$, Alcance $=35,70$ metros e Modelo $=$ Esférico, $R^{2}=0,942$ e $\mathrm{DE}=0,50$ indicando mediana dependência espacial.

Os parâmetros dos semivariogramas cruzados isotrópicos teóricos do $\mathrm{Ln}(\mathrm{Vol})$ para os três tamanhos de amostras e os parâmetros do semivariograma simples isotrópico do Ln (DAP) para a população estão apresentados na Tabela 4. Observa-se que para o Ln (Vol) os patamares diminuíram com a diminuição do número de amostras, o mesmo acontecendo com o efeito pepita, o alcance não foi influenciado pela variação no número de amostras. $\mathrm{O} \mathrm{R}^{2}$ também não sofreu influência do número de amostras. 


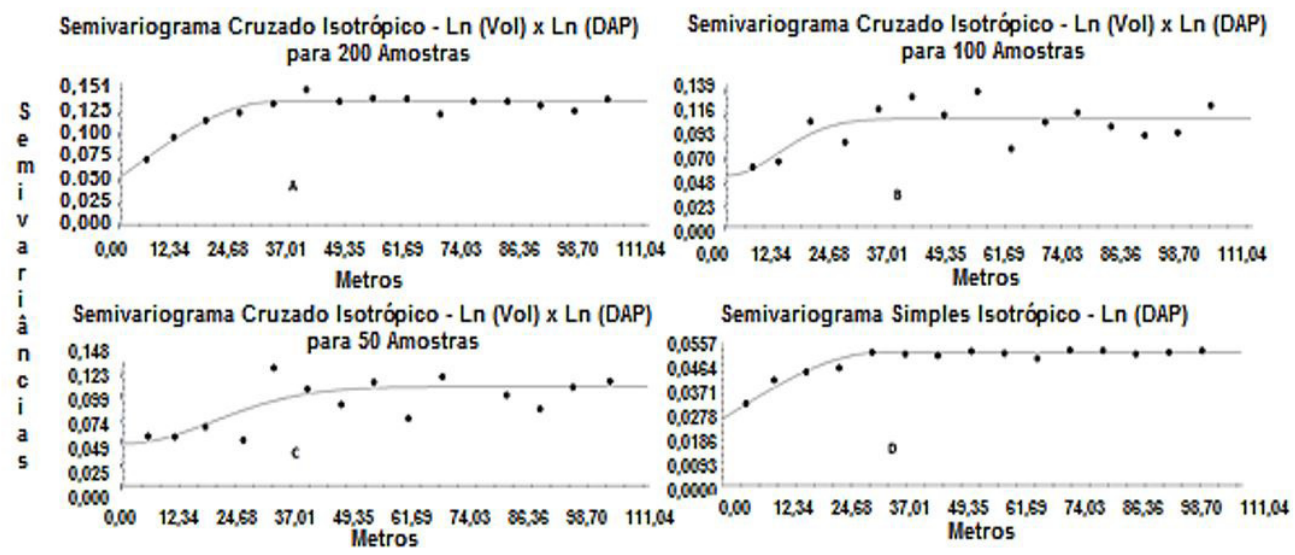

Figura 3. Semivariogramas Cruzados Isotrópicos amostrais e teóricos do Volume [ $\left.\operatorname{Ln}\left(\mathrm{cm}^{3}\right)\right]$ X DAP [Ln (cm)] das amostras de 200 (A), 100 (B), 50 (C) e do DAP (D) Semivariograma Simples Isotrópico da variável secundária DAP. Figure 3. Cross semivariograms sample and theoretical isotropic volume $[\mathrm{Ln}(\mathrm{cm} 3)]$ X DAP [Ln $(\mathrm{cm})]$ of samples of 200 (A), 100 (B), 50 (C) and DAP (D) Semivariogram simple Isotropic PAD secondary variable.

Tabela 4. Parâmetros dos Semivariogramas Isotrópicos Cruzados Ln (Vol) $\times$ Ln (DAP) para as três amostras e semivariograma simples para o Ln (DAP) com 1875 amostras.

Table 4. Parameters of cross semivariograms isotropic $\operatorname{Ln}(\mathrm{Vol}) \times \operatorname{Ln}(\mathrm{DAP})$ for the three samples and simple semivariogram for Ln (DAP) with 1875 samples.

\begin{tabular}{lcccccc}
\multicolumn{1}{c}{ Amostra } & \multicolumn{7}{c}{ Parâmetros dos Semivariogramas } \\
\cline { 2 - 8 } & Mod & Pep & Pat & Alc & $\mathbf{R}^{2}$ & DE \\
\hline $200(\mathrm{Ln}(\mathrm{Vol}) \times \mathrm{DAP})$ & Esf & 0,054 & 0,137 & 33,80 & 0,89 & 0,61 \\
$100(\mathrm{Ln}(\mathrm{Vol}) \times \mathrm{DAP})$ & Gaus & 0,052 & 0,111 & 16,20 & 0,49 & 0,53 \\
\hline $50(\mathrm{Ln}(\mathrm{Vol}) \times \mathrm{DAP})$ & Gaus & 0,046 & 0,108 & 28,20 & 0,49 & 0,57 \\
$1875(\mathrm{Ln}(\mathrm{DAP}))$ & Esf & 0,027 & 0,055 & 35,70 & 0,94 & 0,50 \\
\hline
\end{tabular}

Pep = Efeito Pepita; Mod = Modelo; Esf = Esférico; Gaus = Gaussiano; Pat = Patamar; DE = Dependência Espacial.

\subsection{Construção dos mapas da krigagem e da cokrigagem}

Os mapas da krigagem e da cokrigagem para as três amostras estão apresentados na Figura 4. Mapas do volume de madeira de eucalipto com a krigagem e a cokrigagem também foram construídos por Carvalho et al. (2012). Para a cokrigagem eles utilizaram a compactação do solo e o diâmetro basal da árvore como variáveis secundárias, chegando à conclusão de que essas duas variáveis secundárias tinham forte correlação com o volume de madeira e que também possuíam forte dependência espacial em conjunto com o volume.

Os mapas com 200, 100 amostras apresentam estruturas semelhantes para a krigagem e cokrigagem, estimando volumes com valores bem próximos um do outro, porém os mapas para 50 amostras, apesar de apresentarem visível semelhança, não estimam valores próximos para a mesma localização.

Quando a amostra foi de tamanho 50, naturalmente houve um aumento na distância entre as amostras e a dependência espacial não pôde ser determinada com boa precisão, além disso, aumentou a dificuldade de captação do verdadeiro valor do efeito pepita. Esses fatores juntos podem ter prejudicado a construção do mapa com krigagem. Já no mapa da cokrigagem, as informações faltantes devido ao aumento da distância entre os pontos amostrais do volume foram supridas pela informação da variável secundaria DAP.

Vale ressaltar que nas duas técnicas krigagem e cokrigagem, e para os três tamanhos da amostra, a estrutura espacial do volume foi captada, ou seja, nos locais em que as árvores eram menores ou maiores no seu conjunto, todos os mapas o demarcaram exatamente no mesmo local, variando apenas a dimensão da 

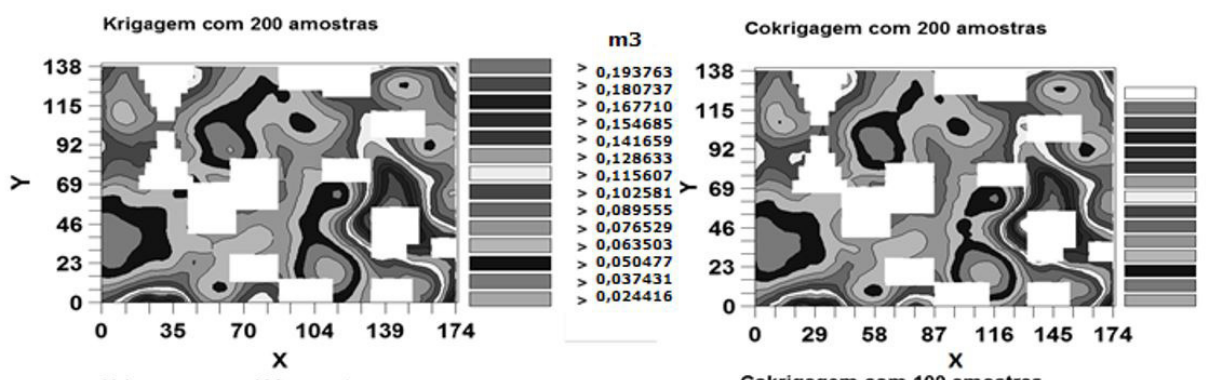

m3

0,190362

0,178554
0,166747
0,154939

0,143132

0,119516

0,107709
0,095901

0,084094

0,072286

0,048671

0,036863
0,025056

Krigagem com 100 amostras

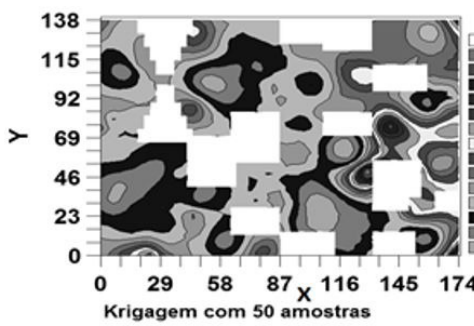

m3

Cokrigagem com 100 amostras

0,253575

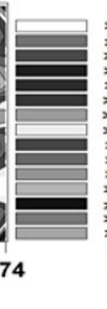

0,237783
0,221991

0,206200
0,190408

0,174617
0,158825

0,158825
0,143034

0,127242

0,095659

0,079867
0,064076

0,064076
0,048284
0,032493

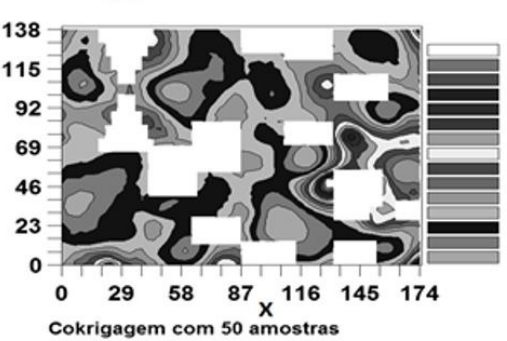

m3

0,241362

0,211753

0,196948
0,182143

0,167338

0,152534
0,137729

0,122924

0,108120
0,093315

0,093315
0,078510

0,048901

0,034093

m3
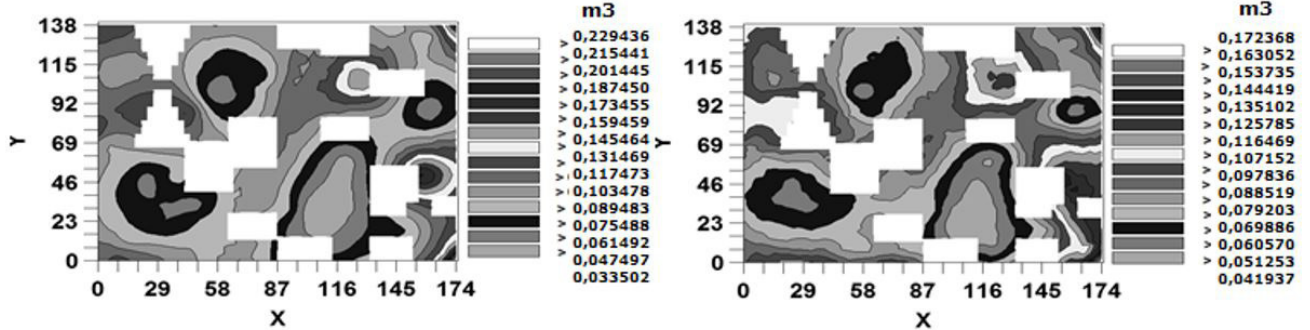

Figura 4. Mapas da krigagem e da cokrigagem com as amostras de 200, 100 e 50.

Figure 4. Maps of kriging and cokriging to the samples 200, 100 and 50.

estimativa do volume para cada um dos seis mapas apresentados. Os erros cometidos pelas duas técnicas estão apresentados na Tabela 5, inesperadamente a diminuição do número de amostras tanto para a krigagem como para a cokrigagem foi acompanhada de uma diminuição no erro da estimativa do volume total. A explicação para o fato necessita de um estudo mais aprofundado sobre todos os aspectos que envolvem a geoestatística.

Os gráficos dos valores observados $\mathrm{x}$ estimados apresentados na Figura 5 mostram que para a krigagem os valores observados menores que $200.000 \mathrm{~cm}^{3}$ foram relativamente bem estimados na grande maioria dos casos para as amostras de 200 e de 100, porém, para a amostra com 50 a precisão é perdida com mais clareza, basta observar o aumento na angulação entre as retas pretas (relação $1 \times 1$ ) e a reta vermelha (tendência linear).

Para a cokrigagem, o comportamento dos pontos foram aparentemente iguais aos da krigagem, comparando tamanho com tamanho da amostra, não sendo possível tomar uma decisão visual de qual dos dois métodos apresentou melhores resultados. É apenas visível que aparentemente as piores estimativas foram realizadas para as amostras de tamanho 50 nos dois métodos.

Observando a Tabela 5 e comparando tamanhos de amostras iguais para verificar quais dos dois métodos apresentou melhor resultado, vemos que para o tamanho 200 todos os dois métodos estimaram a menor o volume total de madeira fornecendo à krigagem uma estimativa melhor com erro percentual de 6,69, erro considerado comum para os padrões de manejo florestal onde os erros de estimativa de volume geralmente estão em torno de 5\% quando usada a regressão. Thaines et al. (2010) compararam sete equações de regressões para estimar o volume de madeira, e o maior erro encontrado por eles na estimativa do volume total foi de $2,2 \%$.

Rocha et al. (2010) compararam dez métodos de estimativas de volume de madeira de eucalipto usados comumente em Ciências Florestais, métodos como: 
Tabela 5. Erros das técnicas krigagem e cokrigagem.

Table 5. Errors of technical kriging and cokriging

\begin{tabular}{|c|c|c|c|c|}
\hline \multirow{2}{*}{ Técnicas } & \multirow{2}{*}{ Parâmetros } & \multicolumn{3}{|c|}{$\mathrm{N}^{\circ}$ amostras } \\
\hline & & 200 & 100 & 50 \\
\hline \multirow{5}{*}{ Krigagem } & ET & $-11,12$ & 2,64 & 1,84 \\
\hline & $\%$ & $-6,69$ & 1,58 & 1,11 \\
\hline & $\mathrm{a}$ & 0,2727 & 0,2730 & 0,1180 \\
\hline & b & 58.579 & 65.900 & 79.185 \\
\hline & $\mathrm{R}^{2}$ & 0,2731 & 0,2447 & 0,0566 \\
\hline \multirow{5}{*}{ Cokrigagem } & ET & $-16,75$ & 8,98 & $-8,48$ \\
\hline & $\%$ & $-10,08$ & 5,41 & $-5,10$ \\
\hline & a & 0,2849 & 0,2974 & 0,1487 \\
\hline & $\mathrm{b}$ & 54.459 & 57.532 & 70.961 \\
\hline & $\mathrm{R}^{2}$ & 0,3402 & 0,3511 & 0,1365 \\
\hline
\end{tabular}

$\mathrm{ET}=$ erro total $\left(\mathrm{m}^{3}\right) ; \mathrm{a}=$ coeficiente angular $; \mathrm{b}=$ intersecção da reta com a ordenada.
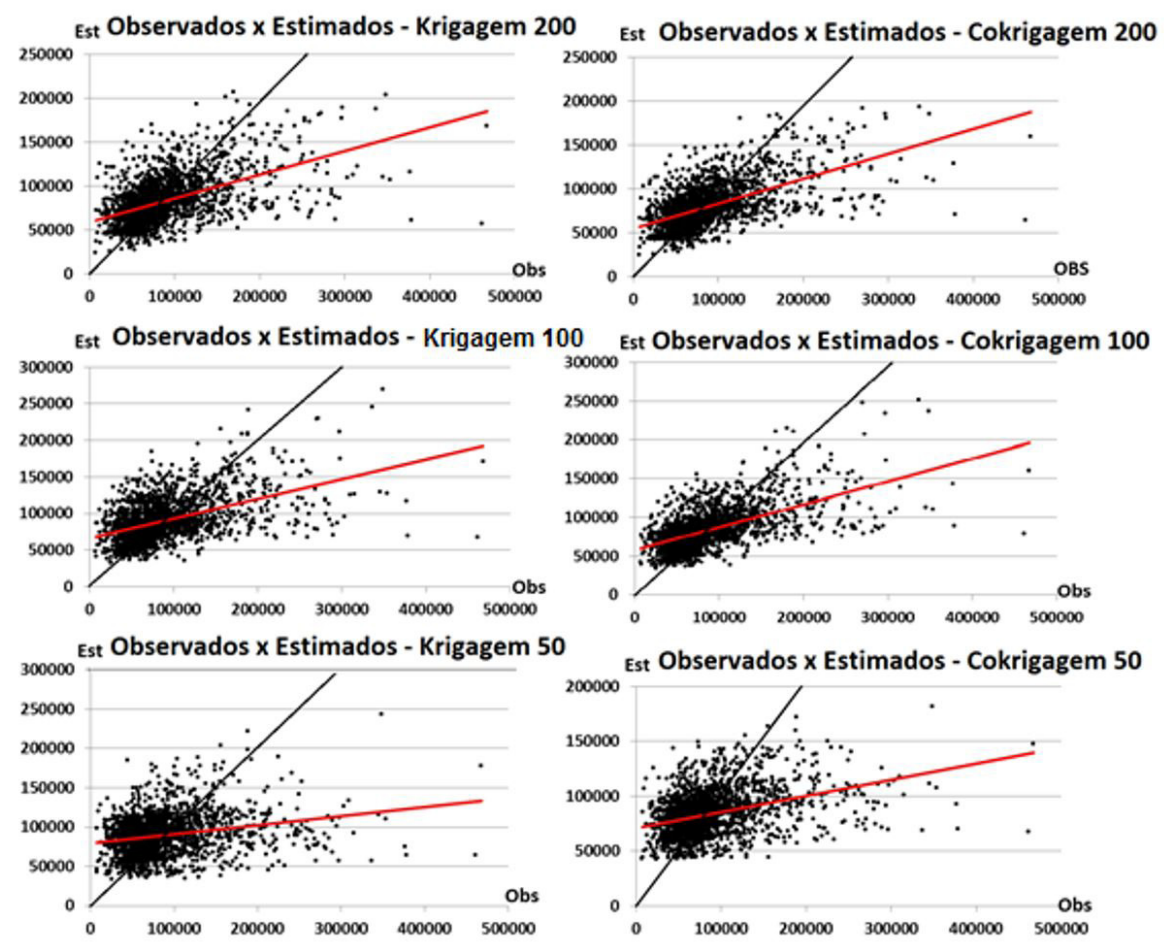

Figura 5. Gráficos dos valores observados $\times$ estimados para a krigagem e cokrigagem nos três tamanhos de amostras. A linha vermelha representa a equação de regressão linear simples, e a linha preta a relação $1 \times 1$ entre os observados e os estimados.

Figure 5. Plots of the observed values $\times$ estimated to kriging and cokriging in the three sample sizes. The red line represents the linear regression equation, and the black line the ratio of $1 \times 1$ between the observed and estimated.

Árvore média, Fator de forma médio, geométrico original etc. e obtiveram erros percentuais diversos, variando entre - $0,22 \%$ para o método árvore média até $72,2 \%$ para o método quociente de forma de Girard por classe. Ainda para a amostra 200, a cokrigagem forneceu melhores resultados para o coeficiente de angulação (a), quanto mais perto do valor 1 (um), mais precisa a estimativa e também para a intersecção $(b)$ que quanto mais próxima de 0 (zero) melhor, além de fornecer maior valor para $\mathrm{R}^{2}$, significando que as estimativas estão mais próximas da verdade do que na krigagem. Para a amostra de tamanho 100 comparando os dois 
métodos, novamente a krigagem estimou melhor o erro total, contudo, a cokrigagem novamente forneceu melhores resultados para o coeficiente angular, para o intercepto e para o $\mathrm{R}^{2}$. Os mesmo resultados ocorreram para a amostra 50.

\section{CONCLUSÃO}

Para a estimação do volume total do povoamento, estranhamente quanto menor o número de amostras, melhores os resultados nos dois métodos.

A cokrigagem forneceu resultados melhores do que a krigagem para estimativas árvore a árvore, indicando que os mapas construídos pela cokrigagem são mais confiáveis do que os da krigagem.

\section{AGRADECIMENTOS}

Os autores agradecem ao IPA - Instituto Agronômico de Pernambuco.

\section{STATUS DA SUBMISSÃO}

Recebido: 4 abr., 2015

Aceito: 26 jun., 2016

\section{AUTOR(ES) PARA CORRESPONDÊNCIA}

\section{Wellington Jorge Cavalcanti Lundgren}

Unidade Acadêmica de Serra Talhada - UAST, Universidade Federal Rural de Pernambuco UFRPE, Avenida Gregório Ferraz Nogueira, s/n, José Tomé de Souza Ramos, CEP 56909-535, Serra Talhada, PE, Brasil e-mail: wellingtonlundgren@yahoo.com.br

\section{REFERÊNCIAS}

Advanced Financial Network - ADVFN. Commodity: madeira [online]. London: ADVFN; 2015 [citado em 2015 abr 4]. Disponível em: advfn.br.com

Barros BC. Volumetria, densidade, carbono e calorimetria de espécies nativas e exóticas no pólo gesseiro do Araripe$P E$ [dissertação]. Recife: Universidade Federal Rural de Pernambuco. 2009.

Biazus A, Hora AB, Leite, B. G. P. Panorama de mercado: painéis de madeira. BNDES Setorial 2010; 32: 49-90.
Bognola IA, Ribeiro PJ, Jr, Silva EAA, Lingnau C, Higa AR. Modelagem uni e bivariada da variabilidade espacial de rendimento de Pinus taeda L. Revista Floresta 2008; 38(2): 463-476.

Carvalho MP, Mendonça VZ, Pereira FCBL, Arf MV, Kappes C, Dalchiavon FC. Produtividade de madeira do eucalipto correlacionada com atributos do solo visando ao mapeamento de zonas específicas de manejo. Ciência Rural 2012; 42(10): 1797-1803. http://dx.doi.org/10.1590/ S0103-84782012005000078.

Cunha AM, Lani JL, Santos GR, Fernandes EI Fo, Trindade FS, Souza E. Espacialização da precipitação pluvial por meio de krigagem e cokrigagem. Pesquisa Agropecuária Brasileira 2013; 48(9): 1179-1191. http://dx.doi.org/10.1590/ S0100-204X2013000900001.

Matheron DF. Traité de geostatistique appliquée. Paris: Technip; 1963.

Mello JM, Batista JLF, Oliveira MS, Ribeiro PJ Jr. Estudo da dependência espacial de características dendrométricas para Eucalyptus grandis. Cerne 2005; 11(2): 113-126.

Mello JM, Diniz FS, Oliveira AD, Mello CR, Scolforo JRS, Acerbi FW Jr. Continuidade espacial para características dendrométricas (numero de fustes e volume) em plantios de eucalyptus grandis. Revista Árvore 2009a; 33(1): 185 194. http://dx.doi.org/10.1590/S0100-67622009000100020.

Mello JM, Diniz FS, Oliveira AD, Scolforo JRS, Acerbi FW Jr, Thiersch CR. Métodos de amostragem e geoestatística para estimativa do número de fustes e volume em plantios de Eucalyptus grandis. Revista Floresta 2009b; 39(1): 157-166.

Mello JM, Oliveira MS, Batista JLF, Justiniano PR Jr, Kanegae H Jr. Uso do estimador geoestatístico para predição volumétrica por talhão. Revista Floresta 2006; 36(2): 251-260.

Mello JM. Geoestatística aplicada ao inventário florestal [tese]. Piracicaba: Escola Superior de Agricultura "Luiz de Queiroz", Universidade de São Paulo; 2004.

Miranda MAS. Evolução do custo de produção e estagnação do preço da madeira. 6. ed. Brasília: CNA Brasil, SENAR, UFV; 2014. p. 1-4. Ativos da Silvicultura, n. 2.

Nogueira FM. B., Bernardi CJ, Couto EG. Aplicação da geoestatística para amostragens de solo em planície de inundação. In: Anais do XXVII Congresso Interamericano de Engenharia Sanitária e Ambiental; 2000; Porto Alegre. Rio de Janeiro: Associação Brasileira de Engenharia Sanitária e Ambiental; 2000.

Paula RC, Pires IE, Borges R, Cruz CD. Predição de ganhos genéticos em melhoramento florestal. Pesquisa Agropecuária Brasileira 2002; 37(2): 159-165. http://dx.doi. org/10.1590/S0100-204X2002000200007.

Rocha TB, Cabacinha CD, Almeida RC, Paula A, Santos RC. Avaliação de métodos de estimativa de volume para 
um povoamento de Eucalyptus urophylla ST Blake no Planalto da Conquista-BA. Enciclopédia Biosfera 2010; 6(10): 1-13.

Souza ZM, Souza GS. Número de amostras e seus efeitos na análise geoestatística e krigagem de atributos do solo. In: Anais do II Simpósio de Geoestatística Aplicada em Ciências Agrárias; 2011; Botucatu. Botucatu: UNESP; 2011.

Thaines F, Braz EM, Mattos PP, Thaines AAR. Equações para estimativa de volume de madeira para a região da bacia do Rio Ituxi, Labréia, AM. Pesquisa Florestal Brasileira 2010; 30(64): 283-289. http://dx.doi.org/10.4336/2010.pfb.30.64.283.
Valverde SR, Soares NS, Silva ML, Jacovine LAG, Neiva S. A. O comportamento do mercado da madeira de eucalipto no Brasil. Biomassa \& Energia 2004; 1(4): 393-403.

Vieira SR. Uso da geoestatística em estudos de variabilidade espacial. In: Universidade Estadual de Campinas - Unicamp. Curso de atualização em conservação do solo. Campinas: Instituto Agronômico de Campinas; 1995.

Yamamoto JK, Landim PMB. Geoestatística: conceitos e aplicações. São Paulo: Oficina de Textos; 2013. 215 p. 